\title{
PLANT STRESS INDUCED BY EXCESSIVE SUCROSE AND AGAR CONCENTRATION ON IN VITRO GERMINATION AND PLANTLET GROWTH OF LAURUS NOBILIS L. (LAURACEAE)
}

\author{
CAvusoglu, A. $.^{1,2^{*}}-$ BOZKURT, D. $^{2}$ \\ ${ }^{1}$ Kocaeli University, Faculty of Agriculture and Natural Sciences, Department of Plant \\ Protection, 41285 Kocaeli, Turkey \\ (e-mail: cavusoglu@kocaeli.edu.tr) \\ ${ }^{2}$ Kocaeli University, Graduate School of Natural and Applied Sciences, Department of \\ Horticulture, 41001 Kocaeli, Turkey \\ (e-mail:deniz.bozkurt@kocaeli.edu.tr) \\ ${ }^{*}$ Corresponding author \\ e-mail: cavusoglu@kocaeli.edu.tr; phone+90-543-844-5323; fax: +90-351-3283 \\ (Received $10^{\text {th }}$ Jul 2020; accepted $17^{\text {th }}$ Sep 2020)
}

\begin{abstract}
Laurus nobilis L. a member of Lauraceae family, is a beautiful medicinal and aromatic, evergreen plant. In this study shortening of in vitro germination time and the appropriate sucrose and agar concentration for this approach were investigated. Excessive sucrose concentration negatively affected germination percentage, root length, shooting percentage and shoot length in Murashige and Skoog Medium (MS). Medium without sucrose ( $0 \mathrm{~g} / \mathrm{L}$ sucrose) having the highest germination rate (96.3\%), the highest root length per plant $(3.84 \mathrm{~cm})$, the highest shooting rate $(96.3 \%)$ and the highest shoot length per plant $(1.68 \mathrm{~cm})$ in all concentrations of sucrose used $(0,10,20$ and $30 \mathrm{~g} / \mathrm{L})$ after 4 weeks of culturing. Although sucrose and agar interactions exerted effects on the germination percentage, additionally all concentrations of agar used $(3,6$ and $9 \mathrm{~g} / \mathrm{L})$ showed that adding excessive amounts to the growth medium was not necessary to induce germination and shooting in general. $0 \mathrm{mg} / \mathrm{L}$ sucrose and $3 \mathrm{~g} / \mathrm{L}$ agar found optimum composition of the media for both rooting and shooting in all used media. This protocol will be useful for rapid and economic large-scale in vitro cultivation of Laurus nobilis to obtain aseptic seedlings. Keywords: recalcitrant seed culture, tissue culture, organic additives, medium specification, rooting, shooting
\end{abstract}

\section{Introduction}

Developing an efficient and rapid system for in vitro tissue and organ culture is highly dependent on plant genus, species, physical and chemical culture environment. The content of culture media for germination, shooting, rooting and multiplication has an enourmous efficacy on costs in large-scale commercial manufacturers producing ornamental plants, vegetable seedling, fruit sapling and secondary metabolites as well as governmental or private scientific research and development centers.

During the past decades different media have been developed for in vitro plant culture. One of the known important components are carbon sources such as sucrose. Sucrose is mostly used 2-4\% to bring in the young explant ready to use carbon (Gamborg et al., 1976). Especially 3\% concentration is choosen in lots of studies as known recommendation of (Murashige and Skoog, 1962). In some cases high level of sucrose can be caused decreasing in vitro germination or the other growth parameters (Jo et al., 2009; Huh et al., 2016). The second known components are gelling agents such as agar. Generally, if solid media is aimed to use, agar is added 0.6-0.8\% ratio to increase media viscosity (Debergh, 1983). In some cases increasing ratio of the gelling agent led to a progressive decrease in 
adventitious shoot per explant (Owens and Wozniak, 1991; Casanova et al., 2008). On the contrary, decreasing ratio of the agent cause turning media solid to liquid is not suitable in some conditions such as germination, rooting or shoot multiplication in some plants. Because of inability of the explant to hold on the surface and subside to the bottom cause undesired physical condition as non-stability of the explant and oxygen deficiency for the plant parts. The model plants for the study, bay laurel (Laurus nobilis L.) belongs to the family Lauraceae family which include valuable genus (Werff and Richter, 1996; Judd et al., 1999; Marques, 2001). Bay laurel are dioecious, evergreen tree or shrub known as laurel, bay laurel or sweet bay (Marzouki et al., 2009). Nearly all plant parts have been used as medicinal-aromatic, ornamental, plant-animal health and environmental purpose for a long time (Patrakar et al., 2012; Chalal et al., 2017). Active chemicals of most of these usage obtained from traditionally grown or naturally existing plants. The high economic value of the species in Lauraceae family has caused these to be destroyed in the natural habitats over years as our long term observations. Optimized tissue culture techniques can provide protection of natural habitats. There are a few studies for different purposes given some information the in vitro propagation of Laurus nobilis L. (Rady et al., 1999; Souayah et al., 2002; Al-Gabbiesh et al., 2014; Nadarajan and Pritchard, 2014; Royandazagh, 2019). The objective of the present study was to evaluate the effect of sucrose and agar concentrations on in vitro germination, primary root and primary shoot growth of bay laurel (L. nobilis) in in vitro seedlings to achieve scientific and economic gain.

\section{Materials and Methods}

The research was carried out at Kocaeli University, Plant Tissue Culture and Biotechnology Laboratory during 2018-2019. The fruits of laurel were picked up from only one tree that have been observing for many years, at natural habitats of Kocaeli City in Turkey. As soon as the bay laurel fruits were collected at 2018 November, 24; they were brought to the laboratory and the fruit flesh was peeled with the help of paper towel. One day after this procedure, the seed coats were removed with fingernail to obtain naked seed. The naked seed (the average weight of 100 naked seeds was $0.574 \mathrm{~g} / \mathrm{seed}$ ) kept in refrigerator $\left(8^{\circ} \mathrm{C}\right)$ until the next day to use. Seeds were washed with running tap water for 1 hour and soaked for $40 \mathrm{~min}$. in $20 \%$ commercial bleach (Na-hypochloride $5 \leq$ ) solution and rinsed in two changes of sterile distilled water. Seeds were cultured on MS medium (Murashige and Skoog, 1962) with full micro, macro elements and vitamins on four trade mark sucrose $\left(\mathrm{C}_{12} \mathrm{H}_{22} \mathrm{O}_{11}-\mathrm{MW}: 342.29\right)(0,10,20$ and $30 \mathrm{~g} / \mathrm{L})$ and three trade mark agaragar concentration $(3,6$ and $9 \mathrm{~g} / \mathrm{L})$. The $\mathrm{pH}$ of the medium was adjusted to 5.6 with $1 \mathrm{M}$ $\mathrm{KOH}$ or $1 \mathrm{M} \mathrm{HCl}$ prior to autoclaving for $15 \mathrm{~min}$. at $121{ }^{\circ} \mathrm{C}$. The media were filled in sterile glass petri plates in $6 \mathrm{~cm}$ diameter subsequently sterile-naked seeds were placed on the surface of media and kept in culture room at $23{ }^{\circ} \mathrm{C}$ under dark condition for four weeks. Each experimental treatment combination consisted of two factors; sucroseXagar (4X3). The experiment laid out in completely randomised design with 3 replications. In only one repeat which was carried out with 3 glass petri dishes each of which contained only one seed. Results were evaluated weekly after cultures along four weeks. The recorded parameters were germination percentages $(\%)$, root length $(\mathrm{cm})$, shooting percentages $(\%)$ and shoot length $(\mathrm{cm})$ of the newly germinating seeds. The data were subjected to analysis of variance and significant differences among the treatments were tested using two-way ANOVA and means were separated by Duncan Multiple Range Test at $\mathrm{P} \leq 0.05$. 


\section{Results and Discussion}

Germination started as of the first week and negative effect of high sucrose concentration observed and statistically detected by the time. There was no positive or negative effect of agar concentration on germination percentage along 4 weeks and at the end of study (Tables 1, 2, 3, 4; Fig. 1). The combination of sucrose and agar in used MS medium significantly affected in vitro seed germination percentage by the second week. At the same time root length was also statistically and negatively affected by high sucrose concentration at the end of the experiment.

Table 1. Laurus nobilis L. germination percentage and root length at the end of the $1^{\text {st }}$ week in the modified MS medium

\begin{tabular}{|c|c|c|c|c|c|c|c|c|c|}
\hline \multicolumn{5}{|c|}{$\begin{array}{l}\text { Germination Percentage (\%) } \\
\text { at the end of the 1st week }\end{array}$} & \multicolumn{5}{|c|}{$\begin{array}{c}\text { Root Length }(\mathrm{cm}) \\
\text { at the end of the } 1 \mathrm{st} \text { week }\end{array}$} \\
\hline & $\begin{array}{l}3 \mathrm{~g} / \mathrm{L} \\
\text { Agar }\end{array}$ & $\begin{array}{l}6 \mathrm{~g} / \mathrm{L} \\
\text { Agar }\end{array}$ & $\begin{array}{l}9 \mathrm{~g} / \mathrm{L} \\
\text { Agar }\end{array}$ & $\begin{array}{c}\text { Mean of } \\
\text { Sucrose } * * *\end{array}$ & & $\begin{array}{l}3 \mathrm{~g} / \mathrm{L} \\
\text { Agar }\end{array}$ & $\begin{array}{l}6 \mathrm{~g} / \mathrm{L} \\
\text { Agar }\end{array}$ & $\begin{array}{l}9 \mathrm{~g} / \mathrm{L} \\
\text { Agar }\end{array}$ & $\begin{array}{c}\text { Mean of } \\
\text { Sucrose**** }\end{array}$ \\
\hline $\begin{array}{c}0 \mathrm{~g} / \mathrm{L} \\
\text { Sucrose }\end{array}$ & $33.3^{*}$ & 22.2 & 33.3 & $29.6 \mathrm{~A}$ & $\begin{array}{c}0 \mathrm{~g} / \mathrm{L} \\
\text { Sucrose }\end{array}$ & $0.17 \mathrm{a} * * * *$ & $0.10 \mathrm{ab}$ & $0.10 \mathrm{ab}$ & $0.12 \mathrm{~A}$ \\
\hline $\begin{array}{c}10 \mathrm{~g} / \mathrm{L} \\
\text { Sucrose }\end{array}$ & 22.2 & 11.1 & 33.3 & $22.2 \mathrm{AB}$ & $\begin{array}{c}10 \mathrm{~g} / \mathrm{L} \\
\text { Sucrose }\end{array}$ & $0.13 \mathrm{ab}$ & $0.03 b$ & $0.13 \mathrm{ab}$ & $0.10 \mathrm{AB}$ \\
\hline $\begin{array}{c}20 \mathrm{~g} / \mathrm{L} \\
\text { Sucrose }\end{array}$ & 22.2 & 11.1 & 0.0 & 11.1 AB & $\begin{array}{c}20 \mathrm{~g} / \mathrm{L} \\
\text { Sucrose }\end{array}$ & $0.06 \mathrm{~b}$ & $0.03 b$ & $0.00 \mathrm{~b}$ & $0.03 \mathrm{AB}$ \\
\hline $\begin{array}{c}30 \mathrm{~g} / \mathrm{L} \\
\text { Sucrose }\end{array}$ & 0.0 & 0.0 & 0.0 & $0.0 \mathrm{~B}$ & $\begin{array}{c}30 \mathrm{~g} / \mathrm{L} \\
\text { Sucrose }\end{array}$ & $0.00 \mathrm{~b}$ & $0.00 \mathrm{~b}$ & $0.00 \mathrm{~b}$ & $0.00 \mathrm{~B}$ \\
\hline $\begin{array}{l}\text { Mean of } \\
\text { Agar*** }^{*}\end{array}$ & 19.5 & 11.1 & 16.7 & & $\begin{array}{c}\text { Mean } \\
\text { of } \\
\text { Agar } * *^{2}\end{array}$ & 0.09 & 0.04 & 0.06 & \\
\hline
\end{tabular}

*N.S.; No significant difference in sucroseXagar concentration interaction in germination percentage, **N.S.; No significant difference in agar concentration in germination percentage and in root length, ***Capital letters denote significantly differences in sucrose concentration in germination percentage and in root length, $* * * *$ Lower-case letters denote significantly differences in sucroseXagar concentration interaction in root length

Table 2. Laurus nobilis L. germination percentage and root length at the end of the $2^{\text {nd }}$ week in the modified MS medium

\begin{tabular}{|c|c|c|c|c|c|c|c|c|c|}
\hline \multicolumn{5}{|c|}{$\begin{array}{l}\text { Germination Percentage (\%) } \\
\text { at the end of the } 2 \text { nd week }\end{array}$} & \multicolumn{5}{|c|}{$\begin{array}{l}\text { Root Length }(\mathrm{cm}) \\
\text { at the end of the } 2 \mathrm{nd} \text { week }\end{array}$} \\
\hline & $\begin{array}{l}3 \mathrm{~g} / \mathrm{L} \\
\text { Agar }\end{array}$ & $\begin{array}{l}6 \mathrm{~g} / \mathrm{L} \\
\text { Agar }\end{array}$ & $\begin{array}{l}9 \mathrm{~g} / \mathrm{L} \\
\text { Agar }\end{array}$ & $\begin{array}{c}\text { Mean of } \\
\text { Sucrose }^{* *}\end{array}$ & & $\begin{array}{l}3 \mathrm{~g} / \mathrm{L} \\
\text { Agar }\end{array}$ & $\begin{array}{l}6 \mathrm{~g} / \mathrm{L} \\
\text { Agar }\end{array}$ & $\begin{array}{l}9 \mathrm{~g} / \mathrm{L} \\
\text { Agar }\end{array}$ & $\begin{array}{c}\text { Mean of } \\
\text { Sucrose }^{* *}\end{array}$ \\
\hline $\begin{array}{c}0 \mathrm{~g} / \mathrm{L} \\
\text { Sucrose }\end{array}$ & $88.9 a^{* * *}$ & $100.0 \mathrm{a}$ & $66.6 a b c$ & $85.2 \mathrm{~A}$ & $\begin{array}{c}0 \mathrm{~g} / \mathrm{L} \\
\text { Sucrose }\end{array}$ & $1.23 \mathrm{a} * * *$ & $0.80 \mathrm{bc}$ & $1.25 \mathrm{a}$ & $1.09 \mathrm{~A}$ \\
\hline $\begin{array}{c}10 \mathrm{~g} / \mathrm{L} \\
\text { Sucrose }\end{array}$ & $88.9 a$ & $66.6 a b c$ & $88.9 a$ & $81.5 \mathrm{~A}$ & $\begin{array}{c}10 \mathrm{~g} / \mathrm{L} \\
\text { Sucrose }\end{array}$ & $0.88 \mathrm{ab}$ & $0.46 b c$ & $1.03 \mathrm{ab}$ & $0.79 \mathrm{AB}$ \\
\hline $\begin{array}{c}20 \mathrm{~g} / \mathrm{L} \\
\text { Sucrose }\end{array}$ & 66.6abc & $66.6 a b c$ & 77.7ab & $70.3 \mathrm{~A}$ & $\begin{array}{c}20 \mathrm{~g} / \mathrm{L} \\
\text { Sucrose }\end{array}$ & $0.37 b c$ & $0.56 b c$ & $0.79 b c$ & 0.57 BC \\
\hline $\begin{array}{c}30 \mathrm{~g} / \mathrm{L} \\
\text { Sucrose }\end{array}$ & $22.2 \mathrm{c}$ & $55.5 b c$ & $33.3 b c$ & $37.0 \mathrm{~B}$ & $\begin{array}{c}30 \mathrm{~g} / \mathrm{L} \\
\text { Sucrose }\end{array}$ & $0.13 c$ & $0.85 b c$ & $0.67 b c$ & $0.55 \mathrm{C}$ \\
\hline $\begin{array}{c}\text { Mean } \\
\text { of } \\
\text { Agar* }\end{array}$ & 66.7 & 72.2 & 66.6 & & $\begin{array}{l}\text { Mean of } \\
\text { Agar***** }\end{array}$ & 0.65 B & 0.67 B & $0.94 A$ & \\
\hline
\end{tabular}

*N.S.; No significant difference in agar concentration in germination percentage, $* *$ Capital letters denote significantly differences in sucrose concentration in germination percentage and in root length, ***Lower-case letters denote significantly differences in sucroseXagar concentration interaction in germination percentage and in root length, $* * *$ Capital letters denote significantly differences in agar concentration in root length 
Table 3. Laurus nobilis L. germination percentage and root length at the end of the $3 r d$ week in the modified MS medium

\begin{tabular}{|c|c|c|c|c|c|c|c|c|c|}
\hline \multicolumn{5}{|c|}{$\begin{array}{l}\text { Germination Percentage (\%) } \\
\text { at the end of the 3rd week }\end{array}$} & \multicolumn{5}{|c|}{$\begin{array}{c}\text { Root Length }(\mathrm{cm}) \\
\text { at the end of the 3rd week }\end{array}$} \\
\hline & $\begin{array}{l}3 \mathrm{~g} / \mathrm{L} \\
\text { Agar }\end{array}$ & $\begin{array}{l}6 \mathrm{~g} / \mathrm{L} \\
\text { Agar }\end{array}$ & $\begin{array}{l}9 \mathrm{~g} / \mathrm{L} \\
\text { Agar }\end{array}$ & $\begin{array}{c}\text { Mean of } \\
\text { Sucrose }^{* *}\end{array}$ & & $\begin{array}{l}3 \mathrm{gg} / \mathrm{L} \\
\text { Agar }\end{array}$ & $\begin{array}{l}6 \mathrm{~g} / \mathrm{L} \\
\text { Agar }\end{array}$ & $\begin{array}{l}9 \mathrm{~g} / \mathrm{L} \\
\text { Agar }\end{array}$ & $\begin{array}{c}\text { Mean of } \\
\text { Sucrose }^{* * * * *}\end{array}$ \\
\hline $\begin{array}{c}0 \mathrm{~g} / \mathrm{L} \\
\text { Sucrose }\end{array}$ & $100.0 \mathrm{a}^{* * *}$ & $100.0 \mathrm{a}$ & $88.9 a$ & $96.3 \mathrm{~A}$ & $\begin{array}{c}0 \mathrm{~g} / \mathrm{L} \\
\text { Sucrose }\end{array}$ & 2.96 & 2.24 & 1.9 & 2.37 \\
\hline $\begin{array}{c}10 \mathrm{~g} / \mathrm{L} \\
\text { Sucrose }\end{array}$ & $88.9 a$ & $66.6 a b$ & $100.0 \mathrm{a}$ & 85.2 AB & $\begin{array}{c}10 \mathrm{~g} / \mathrm{L} \\
\text { Sucrose }\end{array}$ & 2.08 & 1.49 & 2.45 & 2.01 \\
\hline $\begin{array}{c}20 \mathrm{~g} / \mathrm{L} \\
\text { Sucrose }\end{array}$ & $66.6 a b$ & $66.6 a b$ & 77.7ab & $70.3 \mathrm{BC}$ & $\begin{array}{c}20 \mathrm{~g} / \mathrm{L} \\
\text { Sucrose }\end{array}$ & 1.52 & 2.44 & 2.28 & 2.08 \\
\hline $\begin{array}{c}30 \mathrm{~g} / \mathrm{L} \\
\text { Sucrose }\end{array}$ & $33.3 b$ & $66.6 a b$ & $66.6 \mathrm{ab}$ & $55.5 \mathrm{C}$ & $\begin{array}{c}30 \mathrm{~g} / \mathrm{L} \\
\text { Sucrose }\end{array}$ & 1.80 & 1.50 & 1.49 & 1.59 \\
\hline $\begin{array}{c}\text { Mean of } \\
\text { Agar* }\end{array}$ & 72.2 & 74.9 & 83.3 & & $\begin{array}{c}\text { Mean of } \\
\text { Agar* }\end{array}$ & 2.09 & 1.92 & 2.03 & \\
\hline
\end{tabular}

*N.S.; No significant difference in agar concentration in germination percentage and in root length or sucroseXagar concentration interaction in root length, **Capital letters denote significantly differences in sucrose concentration in germination percentage, ***Lower-case letters denote significantly differences in sucroseXagar concentration interaction in germination percentage, **** N.S.; No significant difference in sucrose concentration in root length

Table 4. Laurus nobilis L. germination percentage and root length at the end of the 4th week in the modified MS medium

\begin{tabular}{|c|c|c|c|c|c|c|c|c|c|}
\hline \multicolumn{5}{|c|}{$\begin{array}{c}\text { Germination Percentage (\%) } \\
\text { at the end of the 4th week }\end{array}$} & \multicolumn{5}{|c|}{$\begin{array}{l}\text { Root Length }(\mathrm{cm}) \\
\text { at the end of the 4th week }\end{array}$} \\
\hline & $\begin{array}{l}3 \mathrm{~g} / \mathrm{L} \\
\text { Agar }\end{array}$ & $\begin{array}{l}6 \mathrm{~g} / \mathrm{L} \\
\text { Agar }\end{array}$ & $\begin{array}{l}9 \mathrm{~g} / \mathrm{L} \\
\text { Agar }\end{array}$ & $\begin{array}{c}\text { Mean of } \\
\text { Sucrose }^{* * * * *} \\
\end{array}$ & & $\begin{array}{l}3 \mathrm{~g} / \mathrm{L} \\
\text { Agar }\end{array}$ & $\begin{array}{l}6 \mathrm{~g} / \mathrm{L} \\
\text { Agar }\end{array}$ & $\begin{array}{l}9 \mathrm{~g} / \mathrm{L} \\
\text { Agar }\end{array}$ & $\begin{array}{c}\text { Mean of } \\
\text { Sucrose } * * * * *\end{array}$ \\
\hline $\begin{array}{c}\mathrm{g} / \mathrm{L} \\
\text { Sucrose }\end{array}$ & $100.0 \mathrm{a}^{* * *}$ & $100.0 \mathrm{a}$ & $\begin{array}{c}88.9 \\
\mathrm{ab}\end{array}$ & $96.3 \mathrm{~A}$ & $\begin{array}{c}0 \mathrm{~g} / \mathrm{L} \\
\text { Sucrose }\end{array}$ & $4.22 * *$ & 3.93 & 3.37 & $3.84 \mathrm{~A}$ \\
\hline $\begin{array}{c}10 \mathrm{~g} / \mathrm{L} \\
\text { Sucrose }\end{array}$ & 88.9ab & $66.6 a b$ & $\begin{array}{c}100.0 \\
\mathrm{a}\end{array}$ & 85.2 AB & $\begin{array}{c}10 \mathrm{~g} / \mathrm{L} \\
\text { Sucrose }\end{array}$ & 3.70 & 2.91 & 3.76 & $3.46 \mathrm{AB}$ \\
\hline $\begin{array}{c}20 \mathrm{~g} / \mathrm{L} \\
\text { Sucrose }\end{array}$ & $66.6 a b$ & $88.9 \mathrm{ab}$ & $\begin{array}{l}77.7 \\
\mathrm{ab}\end{array}$ & 77.7 AB & $\begin{array}{c}20 \mathrm{~g} / \mathrm{L} \\
\text { Sucrose }\end{array}$ & 2.72 & 3.02 & 3.76 & 3.17 AB \\
\hline $\begin{array}{c}30 \mathrm{~g} / \mathrm{L} \\
\text { Sucrose }\end{array}$ & $44.4 \mathrm{~b}$ & 77.7ab & $\begin{array}{c}66.6 \\
\mathrm{ab}\end{array}$ & $62.9 \mathrm{~B}$ & $\begin{array}{c}30 \mathrm{~g} / \mathrm{L} \\
\text { Sucrose }\end{array}$ & 2.63 & 2.14 & 2.93 & $2.57 \mathrm{~B}$ \\
\hline $\begin{array}{l}\text { Mean of } \\
\text { Agar* }\end{array}$ & 72.2 & 83.3 & 83.3 & & $\begin{array}{c}\text { Mean of } \\
\text { Agar* }\end{array}$ & 3.32 & 3.00 & 3.46 & \\
\hline
\end{tabular}

*N.S.; No significant difference in agar concentration in germination percentage and in root length, **N.S.; No significant difference in sucroseXagar concentration interaction in root length, *** Lowercase letters denote significantly differences in sucroseXagar concentration interaction in germination percentage, $* * * *$ Capital letters denote significantly differences in sucrose concentration in germination percentage and in root length

Shooting started after two weeks of culture and similarly shooting percentage and shoot length statistically affected from high sucrose concentration negatively and sucroseXagar interaction after 4 weeks (Tables 5, 6 7, 8; Fig. 2). Similarly, there were no positive effect of agar concentrations on shooting percentage along 4 weeks of study. Agar doses showed effectiveness on shoot length in $2^{\text {nd }}$ and $3^{\text {rd }}$ week but at the end of the study the effect has disappeared and agar concentrations showed similarity in statistics. 


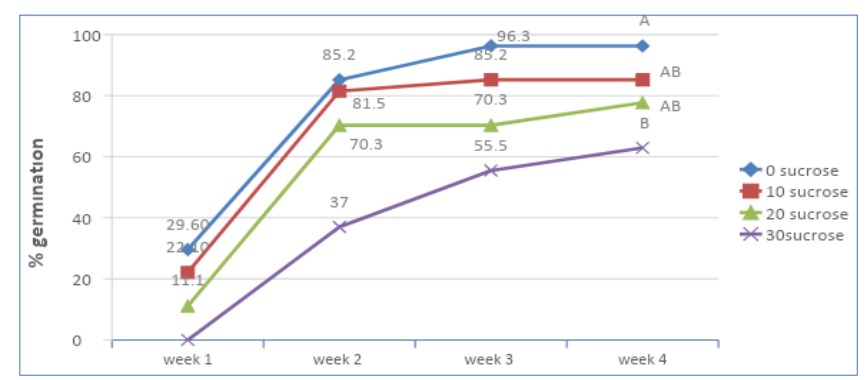

Figure 1. Germination percentage in all sucrose concentrations along 4 weeks

Table 5. Laurus nobilis L. shooting percentage and shoot length at the end of the $1^{\text {st }}$ week in the modified MS medium

\begin{tabular}{|c|c|c|c|c|c|c|c|c|c|}
\hline \multicolumn{5}{|c|}{$\begin{array}{l}\text { Shooting Percentage }(\%) \\
\text { at the end of the 1st week }\end{array}$} & \multicolumn{5}{|c|}{$\begin{array}{l}\text { Shoot Length }(\mathrm{cm}) \\
\text { at the end of the 1st week }\end{array}$} \\
\hline & $\begin{array}{l}3 \mathrm{~g} / \mathrm{L} \\
\text { Agar }\end{array}$ & $\begin{array}{l}6 \mathrm{~g} / \mathrm{L} \\
\text { Agar }\end{array}$ & $\begin{array}{l}9 \mathrm{~g} / \mathrm{L} \\
\text { Agar }\end{array}$ & $\begin{array}{c}\text { Mean of } \\
\text { Sucrose }_{* * *}\end{array}$ & & $\begin{array}{l}3 \mathrm{~g} / \mathrm{L} \\
\text { Agar }\end{array}$ & $\begin{array}{l}6 \mathrm{~g} / \mathrm{L} \\
\text { Agar }\end{array}$ & $\begin{array}{l}9 \mathrm{~g} / \mathrm{L} \\
\text { Agar }\end{array}$ & $\begin{array}{c}\text { Mean of } \\
\text { Sucrose**** }\end{array}$ \\
\hline $\begin{array}{c}\mathbf{0} \mathrm{g} / \mathrm{L} \\
\text { Sucrose }\end{array}$ & $0^{*}$ & 0 & 0 & 0 & $\begin{array}{c}\mathbf{0} \mathrm{g} / \mathrm{L} \\
\text { Sucrose }\end{array}$ & $0^{*}$ & 0 & 0 & 0 \\
\hline $\begin{array}{c}10 \mathrm{~g} / \mathrm{L} \\
\text { Sucrose }\end{array}$ & 0 & 0 & 0 & 0 & $\begin{array}{c}10 \mathrm{~g} / \mathrm{L} \\
\text { Sucrose }\end{array}$ & 0 & 0 & 0 & 0 \\
\hline $\begin{array}{c}20 \mathrm{~g} / \mathrm{L} \\
\text { Sucrose }\end{array}$ & 0 & 0 & 0 & 0 & $\begin{array}{c}20 \mathrm{~g} / \mathrm{L} \\
\text { Sucrose }\end{array}$ & 0 & 0 & 0 & 0 \\
\hline $\begin{array}{c}30 \mathrm{~g} / \mathrm{L} \\
\text { Sucrose }\end{array}$ & 0 & 0 & 0 & 0 & $\begin{array}{c}30 \mathrm{~g} / \mathrm{L} \\
\text { Sucrose }\end{array}$ & 0 & 0 & 0 & 0 \\
\hline $\begin{array}{c}\text { Mean of } \\
\text { Agar** }\end{array}$ & 0 & 0 & 0 & & $\begin{array}{c}\text { Mean of } \\
\text { Agar** }\end{array}$ & 0 & 0 & 0 & \\
\hline
\end{tabular}

*N.S.; No significant difference in sucroseXagar concentration interaction in shooting percentage and shoot length, **N.S.; No significant difference in agar concentration in shooting percentage and shoot length, $* * *$ N.S.; No significant difference in sucrose concentration in shooting percentage and shoot length

Table 6. Laurus nobilis L. shooting percentage and shoot length at the end of the $2^{\text {nd }}$ week in the modified MS medium

\begin{tabular}{|c|c|c|c|c|c|c|c|c|c|}
\hline \multicolumn{5}{|c|}{$\begin{array}{c}\text { Shooting Percentage (\%) } \\
\text { at the end of the } 2 \text { nd week }\end{array}$} & \multicolumn{5}{|c|}{$\begin{array}{c}\text { Shoot Length }(\mathrm{cm}) \\
\text { at the end of the } 2 \mathrm{nd} \text { week }\end{array}$} \\
\hline & $\begin{array}{l}\text { 3g/L } \\
\text { Agar }\end{array}$ & $\begin{array}{l}6 \mathrm{~g} / \mathrm{L} \\
\text { Agar }\end{array}$ & $\begin{array}{l}9 \mathrm{~g} / \mathrm{L} \\
\text { Agar }\end{array}$ & $\begin{array}{c}\text { Mean of } \\
\text { Sucrose } \\
* * * *\end{array}$ & & $\begin{array}{l}3 \mathrm{~g} / \mathrm{L} \\
\text { Agar }\end{array}$ & $\begin{array}{l}6 \mathrm{~g} / \mathrm{L} \\
\text { Agar }\end{array}$ & $\begin{array}{l}\text { 9g/L } \\
\text { Agar }\end{array}$ & $\begin{array}{c}\text { Mean of } \\
\text { Sucrose } \\
* *\end{array}$ \\
\hline $\begin{array}{c}\text { 0 g/L } \\
\text { Sucrose }\end{array}$ & $\begin{array}{c}88.9 a^{* *} \\
*\end{array}$ & $100 \mathrm{a}$ & $66.6 a b c$ & 85.2 A & $\begin{array}{c}0 \mathrm{~g} / \mathrm{L} \\
\text { Sucrose }\end{array}$ & $\begin{array}{l}0.28 \mathrm{ab}^{*} \\
* *\end{array}$ & $0.26 a b$ & $0.37 \mathrm{a}$ & 0.30 \\
\hline $\begin{array}{c}10 \mathrm{~g} / \mathrm{L} \\
\text { Sucrose }\end{array}$ & 77.8ab & $33.3 \mathrm{bc}$ & $88.9 a$ & $66.7 \mathrm{~A}$ & $\begin{array}{c}10 \mathrm{~g} / \mathrm{L} \\
\text { Sucrose }\end{array}$ & $0.27 a b$ & $0.25 \mathrm{ab}$ & $0.32 \mathrm{a}$ & 0.28 \\
\hline $\begin{array}{c}20 \mathrm{~g} / \mathrm{L} \\
\text { Sucrose }\end{array}$ & $66.6 a b c$ & $55.5 \mathrm{abc}$ & $66.6 a b c$ & $62.9 \mathrm{AB}$ & $\begin{array}{c}20 \mathrm{~g} / \mathrm{L} \\
\text { Sucrose }\end{array}$ & $0.18 \mathrm{ab}$ & $0.21 \mathrm{ab}$ & $0.24 \mathrm{ab}$ & 0.21 \\
\hline $\begin{array}{c}30 \mathrm{~g} / \mathrm{L} \\
\text { Sucrose }\end{array}$ & $22.2 \mathrm{c}$ & $55.5 \mathrm{abc}$ & $33.3 \mathrm{bc}$ & $37.0 \mathrm{~B}$ & $\begin{array}{r}30 \mathrm{~g} / \mathrm{L} \\
\text { Sucrose }\end{array}$ & $0.10 \mathrm{~b}$ & $0.18 \mathrm{ab}$ & $0.30 \mathrm{ab}$ & 0.19 \\
\hline $\begin{array}{c}\text { Mean of } \\
\text { Agar* }\end{array}$ & 63.9 & 61.1 & 63.9 & & $\begin{array}{c}\text { Mean } \\
\text { of Agar } \\
* * * * * *\end{array}$ & $0.21 \mathrm{C}$ & $0.23 B$ & $0.31 \mathrm{~A}$ & \\
\hline
\end{tabular}

*N.S.; No significant difference in agar concentration in shooting percentage, **N.S.; No significant difference in sucrose concentration in shoot length, *** Lower-case letters denote significantly differences in sucroseXagar concentration interaction in shooting percentage and shoot length, ***** Capital letters denote significantly differences in sucrose concentration in shooting percentage, *****Capital letters denote significantly differences in agar concentration in shoot length 
Table 7. Laurus nobilis L. shooting percentage and shoot length at the end of the $3^{\text {rd }}$ week in the modified MS medium

\begin{tabular}{|c|c|c|c|c|c|c|c|c|c|}
\hline \multicolumn{5}{|c|}{$\begin{array}{l}\text { Shooting Percentage (\%) } \\
\text { at the end of the 3rd week }\end{array}$} & \multicolumn{5}{|c|}{$\begin{array}{c}\text { Shoot Length }(\mathrm{cm}) \\
\text { at the end of the 3rd week }\end{array}$} \\
\hline & $\begin{array}{l}3 \mathrm{~g} / \mathrm{L} \\
\text { Agar }\end{array}$ & $\begin{array}{l}6 \mathrm{~g} / \mathrm{L} \\
\text { Agar }\end{array}$ & $\begin{array}{l}9 \mathrm{~g} / \mathrm{L} \\
\text { Agar }\end{array}$ & $\begin{array}{c}\text { Mean of } \\
\text { Sucrose } \\
* * *\end{array}$ & & $\begin{array}{l}3 \mathrm{~g} / \mathrm{L} \\
\text { Agar }\end{array}$ & $\begin{array}{l}6 \mathrm{~g} / \mathrm{L} \\
\text { Agar }\end{array}$ & $\begin{array}{l}9 \mathrm{~g} / \mathrm{L} \\
\text { Agar }\end{array}$ & $\begin{array}{c}\text { Mean of } \\
\text { Sucrose } \\
* * *\end{array}$ \\
\hline $\begin{array}{c}\mathrm{0} \mathrm{g} / \mathrm{L} \\
\text { Sucrose }\end{array}$ & $100 a^{*}$ & $100 \mathrm{a}$ & 66.6abc & 88.9 A & $\begin{array}{c}0 \mathrm{~g} / \mathrm{L} \\
\text { Sucrose }\end{array}$ & $\begin{array}{c}0.92 \mathrm{abc} \\
*\end{array}$ & $0.67 b c$ & $1.18 \mathrm{a}$ & $0.92 \mathrm{~A}$ \\
\hline $\begin{array}{l}10 \mathrm{~g} / \mathrm{L} \\
\text { Sucrose }\end{array}$ & 88.9ab & $\begin{array}{c}55.5 b \\
c\end{array}$ & $88.9 \mathrm{ab}$ & $77.8 \mathrm{AB}$ & $\begin{array}{l}10 \mathrm{~g} / \mathrm{L} \\
\text { Sucrose }\end{array}$ & $0.58 \mathrm{c}$ & $0.67 b c$ & $1.12 \mathrm{ab}$ & $0.79 \mathrm{AB}$ \\
\hline $\begin{array}{l}20 \mathrm{~g} / \mathrm{L} \\
\text { Sucrose }\end{array}$ & $\begin{array}{c}66.6 a b \\
c\end{array}$ & $\begin{array}{c}55.5 b \\
c\end{array}$ & $77.7 \mathrm{ab}$ & $66.6 \mathrm{BC}$ & $\begin{array}{l}20 \mathrm{~g} / \mathrm{L} \\
\text { Sucrose }\end{array}$ & $0.43 c$ & $0.59 \mathrm{c}$ & $0.81 \mathrm{abc}$ & $0.61 \mathrm{~B}$ \\
\hline $\begin{array}{c}30 \mathrm{~g} / \mathrm{L} \\
\text { Sucrose }\end{array}$ & & $\begin{array}{c}66.6 \mathrm{a} \\
\mathrm{bc}\end{array}$ & $55.5 \mathrm{bc}$ & $51.8 \mathrm{C}$ & $\begin{array}{c}30 \mathrm{~g} / \mathrm{L} \\
\text { Sucrose } \\
\end{array}$ & $0.50 \mathrm{c}$ & $0.53 \mathrm{c}$ & $0.60 \mathrm{c}$ & 0.54 B \\
\hline $\begin{array}{c}\text { Mean of } \\
\text { Agar** }^{*}\end{array}$ & 72.2 & 69.4 & 72.2 & & $\begin{array}{c}\text { Mean of } \\
\text { Agar***** }\end{array}$ & $0.61 \mathrm{~B}$ & 0.62 B & 0.93 A & \\
\hline
\end{tabular}

* Lower-case letters denote significantly differences in sucroseXagar concentration interaction in shooting percentage and shoot length, ** N.S.; No significant difference in agar concentration in shooting percentage, *** Capital letters denote significantly differences in sucrose concentration in shooting percentage and shoot length, $* * * *$ Capital letters denote significantly differences in agar concentration in shoot length

Table 8. Laurus nobilis L. shooting percentage and shoot length at the end of the 4th week in the modified MS medium

\begin{tabular}{|c|c|c|c|c|c|c|c|c|c|}
\hline \multicolumn{5}{|c|}{$\begin{array}{l}\text { Shooting Percentage }(\%) \\
\text { at the end of the 4th week }\end{array}$} & \multicolumn{5}{|c|}{$\begin{array}{c}\text { Shoot Length }(\mathrm{cm}) \\
\text { at the end of the 4th week }\end{array}$} \\
\hline & $\begin{array}{l}3 \text { g/L } \\
\text { Agar }\end{array}$ & $\begin{array}{l}6 \text { g/L } \\
\text { Agar }\end{array}$ & $\begin{array}{l}9 \text { g/L } \\
\text { Agar }\end{array}$ & $\begin{array}{c}\text { Mean of } \\
\text { Sucrose } \\
* * *\end{array}$ & & $\begin{array}{l}3 \text { g/L } \\
\text { Agar }\end{array}$ & $\begin{array}{l}6 \mathrm{~g} / \mathrm{L} \\
\text { Agar }\end{array}$ & $\begin{array}{l}9 \text { g/L } \\
\text { Agar }\end{array}$ & $\begin{array}{c}\text { Mean of } \\
\text { Sucrose } \\
* * *\end{array}$ \\
\hline $\begin{array}{c}0 \mathrm{~g} / \mathrm{L} \\
\text { Sucrose }\end{array}$ & $100 a^{*}$ & $100 \mathrm{a}$ & 88.9ab & $96.3 \mathrm{~A}$ & $\begin{array}{c}0 \mathrm{~g} / \mathrm{L} \\
\text { Sucrose }\end{array}$ & $1.87 \mathrm{a}^{*}$ & $1.40 \mathrm{abcd}$ & $1.77 \mathrm{ab}$ & $1.68 \mathrm{~A}$ \\
\hline $\begin{array}{c}10 \mathrm{~g} / \mathrm{L} \\
\text { Sucrose }\end{array}$ & $88.9 \mathrm{ab}$ & $55.5 \mathrm{bc}$ & 88.9ab & $77.8 \mathrm{AB}$ & $\begin{array}{c}10 \mathrm{~g} / \mathrm{L} \\
\text { Sucrose }\end{array}$ & $1.28 \mathrm{abcd}$ & $1.60 \mathrm{abc}$ & $1.65 \mathrm{ab}$ & $1.51 \mathrm{~A}$ \\
\hline $\begin{array}{c}20 \mathrm{~g} / \mathrm{L} \\
\text { Sucrose }\end{array}$ & 66.6abc & 77.7ab & 77.7ab & 74.0 BC & $\begin{array}{c}20 \mathrm{~g} / \mathrm{L} \\
\text { Sucrose }\end{array}$ & $0.77 \mathrm{c}$ & $0.72 \mathrm{c}$ & $1.37 \mathrm{abcd}$ & 0.95 B \\
\hline $\begin{array}{c}30 \mathrm{~g} / \mathrm{L} \\
\text { Sucrose }\end{array}$ & $33.3 \mathrm{c}$ & 66.6abc & 66.6abc & $55.5 \mathrm{C}$ & $\begin{array}{c}30 \mathrm{~g} / \mathrm{L} \\
\text { Sucrose }\end{array}$ & $0.83 \mathrm{c}$ & $0.89 \mathrm{~cd}$ & $1.05 \mathrm{bcd}$ & 0.93 B \\
\hline $\begin{array}{l}\text { Mean of } \\
\text { Agar** }\end{array}$ & 72.2 & 75.0 & 80.5 & & $\begin{array}{l}\text { Mean of } \\
\text { Agar** }\end{array}$ & 1.19 & 1.15 & 1.46 & \\
\hline
\end{tabular}

* Lower-case letters denote significantly differences in sucroseXagar concentration interaction in shooting percentage and shoot length, ** N.S.; No significant difference in agar concentration in shooting percentage and shoot length, $* * *$ Capital letters denote significantly differences in sucrose concentration in shooting percentage and shoot length

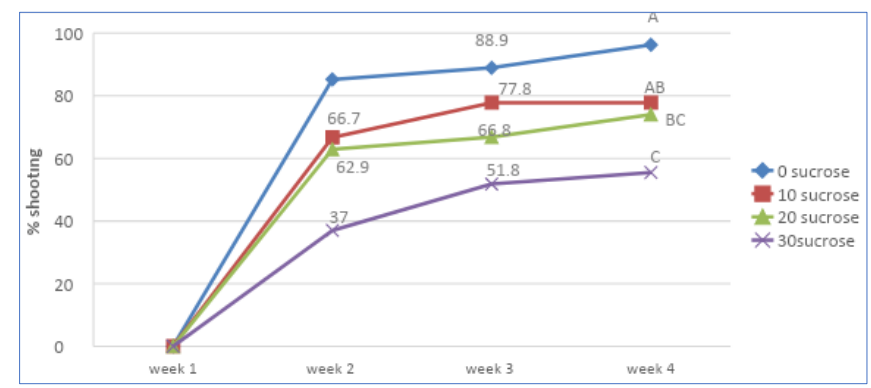

Figure 2. Shooting percentage in all sucrose concentrations along 4 weeks 
Studies are available outlining the ineffectiveness of excessive agar or sucrose doses in in vitro cultures. For example; Rabaiolli et al. (2017) demonstrated better in vitro rhizogenesis was achieved when $1 / 2$ WPM medium supplemented with $30 \mathrm{~cm}^{3}$ vermiculite without agar than $3.5 \mathrm{~g} / \mathrm{L}$ or $7 \mathrm{~g} / \mathrm{L}$ used agar for primary and secondary root percentages in in vitro and survival rate and leaf number in ex vitro in Handroanthus chrysotrichus. Suthar et al. (2011), in their work on Boswellia serrata in in vitro, studied agar at the rate of $0.0 ; 0.2 ; 0.4 ; 0.6 ; 0.8 \% \mathrm{w} / \mathrm{v}$ in $\mathrm{MS}+0.5 \mathrm{mg} / \mathrm{L} \mathrm{BAP}+0.05 \mathrm{mg} / \mathrm{L}$ NAA for shoot multiplication from shoot clusters and 0.0 to $1 \%(\mathrm{w} / \mathrm{v})$ agar in rooting medium containing $0.5 \mathrm{mg} / \mathrm{L} \mathrm{IBA}+0.25 \mathrm{mg} / \mathrm{L} \mathrm{NAA}+$ antioxidants solution from rooting from shoots. According to their results $0.2 \%$ agar concentration was the best in number of shoots and $0.0 \%$ agar gave the highest rate of shoot length, number of leaves, fresh and dry weight, chlorophyll a, b and total. Similarly, they found that $0.0 \%$ agar gave the highest rooting percentage, number of roots, root length and shoot length. Casanova et al. (2008) studied on agar concentration $\left(0,2,4,6,8,10\right.$ and $\left.12 \mathrm{~g} \mathrm{dm}^{3}\right)$ and vessel closure for Dianthus caryophyllus in vitro culture. They emphasized that the highest organogenic response was obtained in MS medium solidified with $2 \mathrm{~g} \mathrm{dm}^{3}$ agar (17.7 shoots per petal) and the least response was obtained in MS medium with $12 \mathrm{~g} \mathrm{dm}^{3}$ (4.3 shoots per petal) after 30 days of culture. Cortés-Olmos et al. (2018) studied on different sucrose (20 and $\left.30 \mathrm{gL}^{-1}\right)$ and agar $\left(8\right.$ and $\left.10 \mathrm{gL}^{-1}\right)$ in full and half strength MS medium for Lophophora williamsii. They found that neither agar and sugar concentrations nor MS strength changed seed germination percentage after 49 days. But seedling size and areoles per seedling found higher in lower sucrose $\left(20 \mathrm{gL}^{-1}\right)$ and lower agar $\left(8 \mathrm{gL}^{-1}\right)$ than higher ones $\left(30 \mathrm{gL}^{-1}\right.$ sucrose and $10 \mathrm{gL}^{-1}$ agar). In another study, Gürel and Gülşen (1998) studied on two cultivars of Prunus amygdalus shoot tip in vitro culture in MS media. Sucrose concentrations were 2, $3,4,5$ and $6 \%$ at $0.7 \%$ agar and agar were examined at the rate of $0.5 ; 0.6 ; 0.7 ; 0.8$ and $0.9 \%$ at $3 \%$ sucrose. They emphasized that not in initiation stage but both multiplication and transplantation stage highest and lowest sucrose levels negatively affected shoot production and growth rate of developing shoots. 3 and $4 \%$ sucrose level found better in this stages. In addition, they found that the increasing concentration of agar caused a decrease in the growth of shoot tip explants in initiation stage $0.5 ; 0.6$ and $0.7 \%$ agar were found significantly better than 0.8 and 0.9 agar on shoot development. Schulze et al. (2017) studied on in vitro germination of Prunus lusitanica. They used MS media with or without $\mathrm{GA}_{3}$ and with different BA with two sucrose level (30 and $\left.60 \mathrm{gL}^{-1}\right)$. Their findings showed that radical and shoot emergence were greater on media with $30 \mathrm{gL}^{-1}$ sucrose than with $60 \mathrm{gL}^{-1}$. In Hancornia speciosa cultured in vitro, dos Santos et al. (2017) observed that increasing sucrose concentration up to $60 \mathrm{~g} \mathrm{~L}^{-1}$ reduced germination speed and seedling height when they use $\left(15,30,45\right.$ and $60 \mathrm{~g} \mathrm{~L}^{-1}$ sucrose) after 60 days of in vitro culturing of the naked embryos.

\section{Conclusion}

In conclusion, the results of this study revealed that increasing sucrose concentration as carbon source negatively influence germination efficiency of Laurus nobilis from naked seeds derived from mature female tree (Fig. 3). Among all the treatments, all parameters recorded to evaluate seedling growth of Laurus nobilis showed better performance in $0 \mathrm{~g} / \mathrm{L}$ of sucrose than 10,20 , and $30 \mathrm{~g} / \mathrm{L}$ in $\mathrm{MS}$ medium. Higher concentration of sucrose caused lateness in germination and shooting with reduced germination and shooting rate, root and shoot length (Figs. 4, 5, 6, 7). When sucrose doses 
are ignored and only agar doses are considered, there were no differencies in all parameters at the end of the experiment excepting middle stage of experiment. When sucrose and agar interaction were examined, $0 \mathrm{~g} / \mathrm{L}$ sucrose with $3 \mathrm{~g} / \mathrm{L}$ agar and $0 \mathrm{~g} / \mathrm{L}$ sucrose with $6 \mathrm{~g} / \mathrm{L}$ agar were found $100 \%$ in both germination and shooting percentage than $9 \mathrm{~g} / \mathrm{L}$ agar. This results supports the hypothesis that agar as viscosity source and sucrose as carbon source reduces the availability of nutrients in media to the plants. Moreover, root and shoot length found the highest in $0 \mathrm{~g} / \mathrm{L}$ sucrose with $3 \mathrm{~g} / \mathrm{L}$ agar among all the treatments $(0,10,20$, and $30 \mathrm{~g} / \mathrm{L}$ sucrose with 3,6 and $9 \mathrm{~g} / \mathrm{L}$ agar $)$. The decreased germination and seedling capacity in seed explants may result from a decrease in the amount of water available in the medium that have high rate of sucrose and agar. In this perspective, it must be underlined sucrose and agar compositions should be experienced in all plant genus and explant types for commercially or academically valuable application to lower the cost and to achieve the right results from other tested treatments.

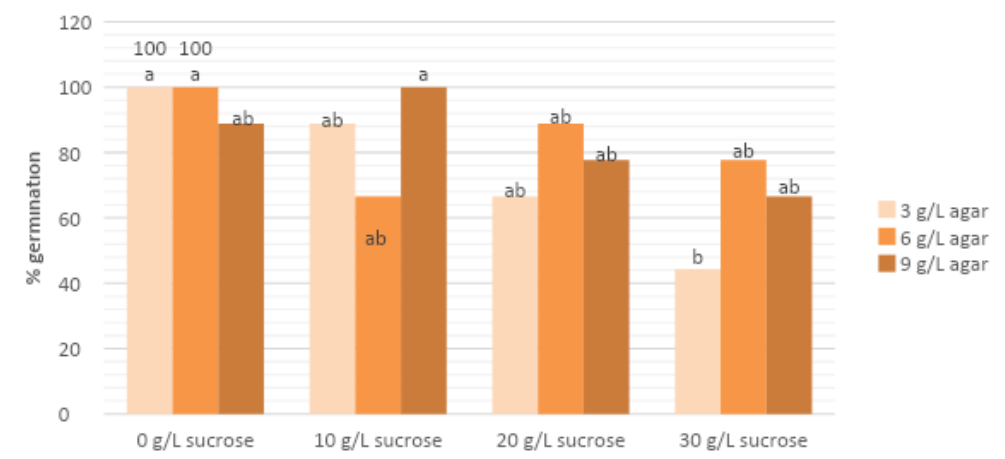

Figure 3. Germination percentage in all sucrose and agar concentrations at the end of the $4^{\text {th }}$ week

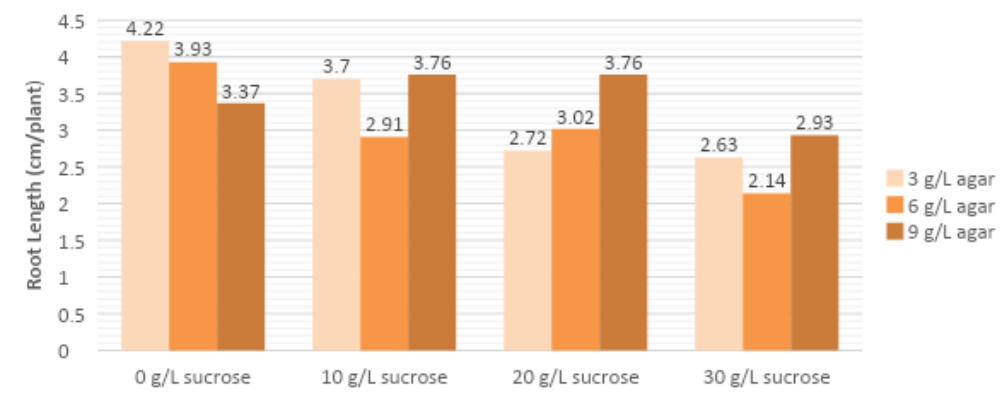

Figure 4. Root length per plant in all sucrose and agar concentrations at the end of the $4^{\text {th }}$ week

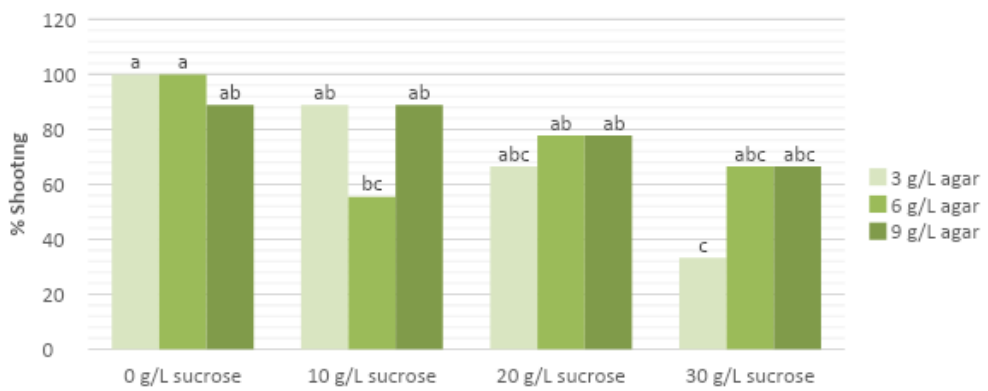

Figure 5. Shooting percentage in all sucrose and agar concentrations at the end of the $4^{\text {th }}$ week 


$$
-7453-
$$

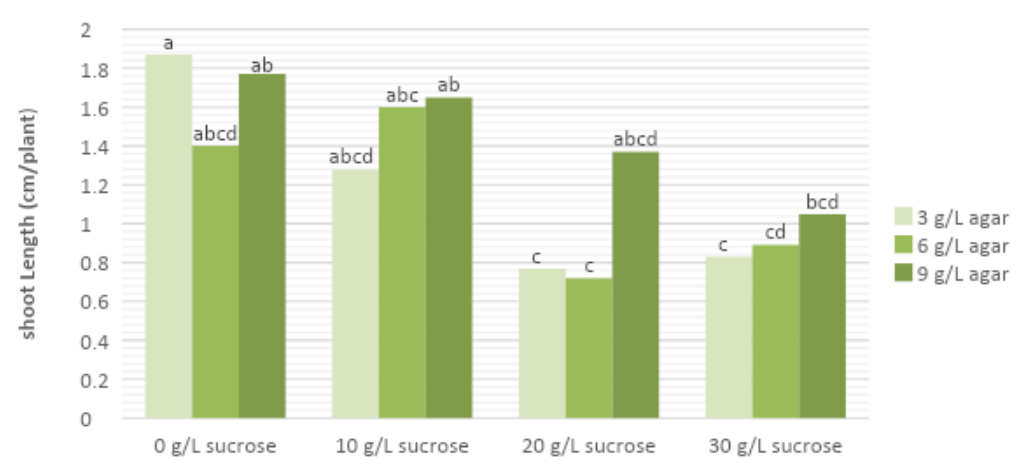

Figure 6. Shoot length per plant in all sucrose and agar concentrations at the end of the $4^{\text {th }}$ week
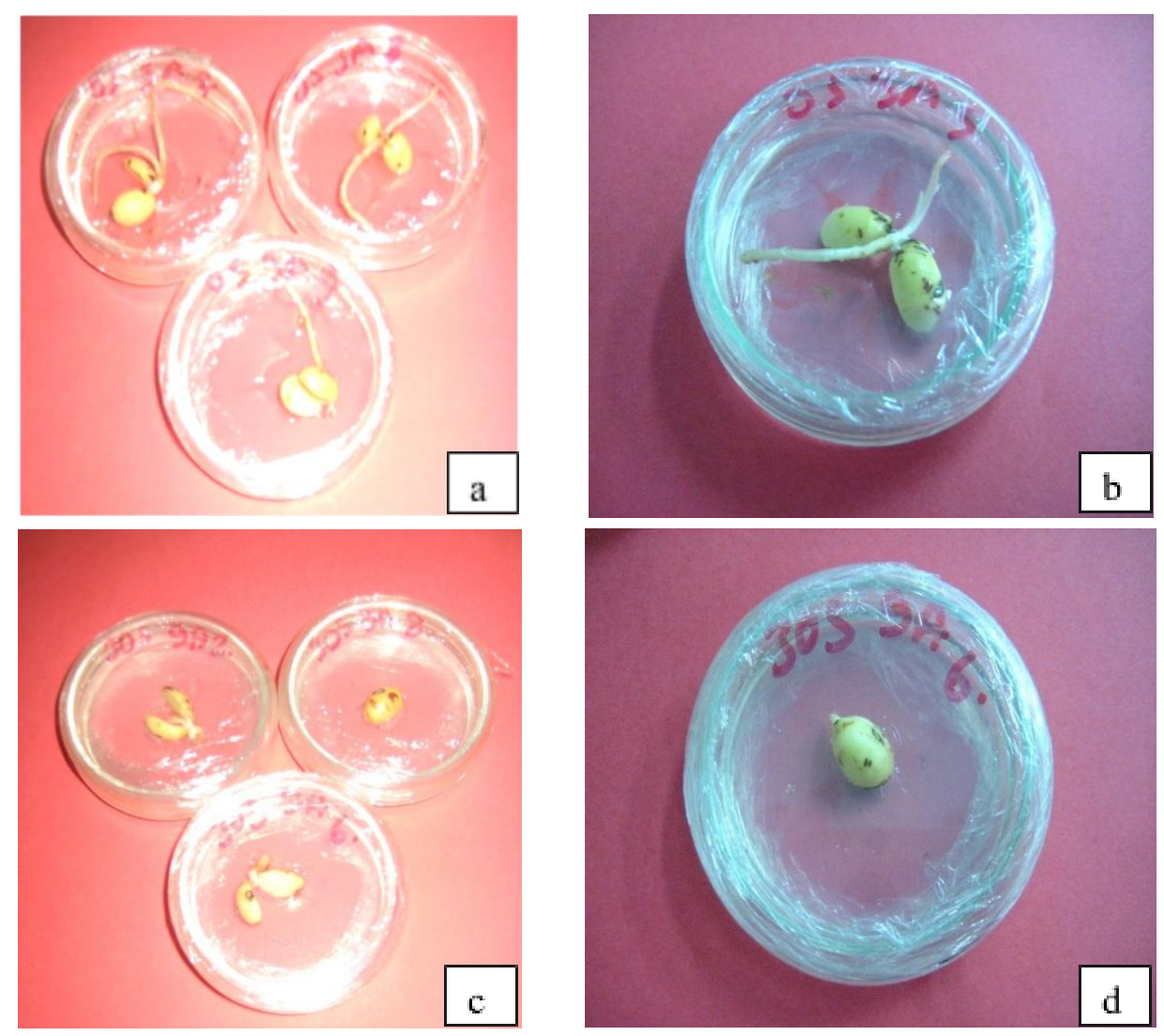

Figure 7. Gemination and shooting of Laurus nobilis L. in different sucrose and agar concentration after 4 weeks; $(a, b)$ successful germination and shooting at $0 \mathrm{~g} / \mathrm{L}$ sucrose and 3 $\mathrm{g} / \mathrm{L}$ agar, $(c, d)$; poor germination and shooting at $30 \mathrm{~g} / \mathrm{L}$ sucrose and $3 \mathrm{~g} / \mathrm{L}$ agar

\section{REFERENCES}

[1] Al-Gabbiesh, A. H., Ghabeish, I. M., Kleinwachter, M., Selmar, D. (2014): Plant regeneration throught somatic embryogenesis from calli derived from leaf bases of Laurus nobilis L.(Lauraceae). - Plant Tissue Cult. \& Biotech. 24: 213-221.

[2] Casanova, E., Moysset, L., Trillas, M. I. (2008): Effects of agar concentration and vessel closure on the organogenesis and hyperhydricity of adventitious carnation shoots. Biologia Plantarum 52: 1-8. 
[3] Chalal, K. K., Kaur, M., Bhardwaj, U., Singla, N., Kaur, A. (2017): A review on chemistry and biological activities of Laurus nobilis L. essential oil. - Journal of Pharmacognosy and Phytochemistry 6: 1153-1161.

[4] Cortés-Olmos, C., Gurrea-Ysasi, G., Prohens, J., Rodríguez-Burruezo, A., Fita, A. (2018): In vitro germination and growth protocols of the ornamental Lophophora williamsii (Lem.) Coult. as a tool for protecting endangered wild populations. - Scientia Horticulturae 237: 120-127.

[5] Debergh, P. C. (1983): Effects of agar brand and concentration on the tissue culture medium. - Physiologia Plantarum 59: 270-276.

[6] dos Santos, M. P., de Aguiar, R. A., Brandão, D. C., Pires, L. L., de Oliveira-Castro, Y., Silva, F. G., da Silva-Neri, L. M., Pereira, D. R. M., de Castro, J. R., Seleguini, A. (2017): Effect of seed desiccation and sucrose concentration on the in vitro establishment of mangabeira (Hancornia speciosa Gomes var. gardneri) seedlings. - African Journal of Agricultural Research 12: 348-353.

[7] Gamborg, O. L., Murashige, T., Thorpe, T. A., Vasil, I. K. (1976): Plant tissue culture media. - In Vitro 12: 473-478.

[8] Gürel, S., Gülşen, Y. (1998): The effects of different sucrose, agar and $\mathrm{pH}$ levels on in vitro shoot production of almond (Amygdalus communis L.). - Turkish Journal of Botany 22: 363-373.

[9] Huh, Y. S., Lee, J. K., Nam, S. Y., Hong, E. Y., Paek, K. Y., Son, S. W. (2016): Effects of altering medium strength and sucrose concentration on in vitro germination and seedling growth of Cypripedium macranthos Sw. - Journal of Plant Botechnol. 43: 132-137.

[10] Jo, E. A., Tewari, R. K., Hahn, E. J., Paek, K. Y. (2009): In vitro sucrose concentration affects growth and acclimatization of Alocasia amazonica plantlets. - Plant Cell Tiss Organ Cult 96: 307-315.

[11] Judd, W. S., Campbell, C. S., Kellog, E. A., Stewens, P. F. (1999): Plant systematics: a phylogenetic approach. - Sinauer Associates, Sunderland.

[12] Marques, C. A. (2001): Anatomia foliar aplicada a taxonomia de especies de Lauraceae Lind. - Universidade Federal de Viçosa, Viçosa.

[13] Marzouki, H., Nasri, N., Jouaud, B., Bonnet, C., Khaldi, A., Bouzid, S., Fady, B. (2009): Population genetic structure of Laurus nobilis L. inferred from transferred nuclear microsatellites. - Silvae Genet. 58: 270-276.

[14] Murashige, T., Skoog, F. (1962): A revised medium for rapid growth and bioassays with tobacco tissue cultures. - Physiol. Plant. 15: 473-497.

[15] Nadarajan, J., Pritchard, H. W. (2014): Biophysical characteristics of successful oilseed embryo cryoprotection and cryopreservation using vacuum infiltration vitrification: an innovation in plant cell preservation. - Plos one 9: e96169.

[16] Owens, L. D., Wozniak, C. A. (1991): Measurement and effects of gel matric potential and expressibility on production of morphogenic callus by cultured sugar beet leaf discs. - Plant Cell Tiss Organ Cult 26: 127-133.

[17] Patrakar, R., Mansuriya, M., Patil, P. (2012): Phytochemical and pharmacological review on Laurus nobilis. - International Journal of Pharmaceutical and Chemical Sciences 1: 595602.

[18] Rabaiolli, S. M. S., Reiniger, L. R. S., Stefanel, C. M., Silva, K. B., Paim, A. F., Ziegler, A. C. F. (2017): Agar does not affect in vitro rhizogenesis and ex vitro acclimatization of Handroanthus chrysotrichus. - Cerne 23: 185-192.

[19] Rady, M. R., Youssef, A. A. (1999): Comparison of essential oils and fats from in vitro cultures and field collected material of Laurus nobilis. - J. Agric. Sci. Mansoura Uni. Egypt 24: 3401-3412.

[20] Royandazagh, D. S. (2019): Potential of flow cytometry in sex determination and in vitro micropropagation of Laurus nobilis L. - Appl. Ecol. Environ. Res. 17: 5953-5964.

[21] Schulze, J. A., Lattier, J. D., Contreras, R. N. (2017): In vitro germination of immature Prunus lusitanica seed. - HortScience 52: 1122-1124. 
[22] Souayah, N., Khouja, M. L., Khaldi, A., Rejeb, M. N., Bouzid, S. (2002): Breeding improvement of Laurus nobilis L. by conventional and in vitro propagation techniques. Journal of Herbs, Spices \& Medicinal Plants 9: 101-105.

[23] Suthar, R. K., Habibi, N., Purohit, S. D. (2011): Influence of agar concentration and liquid medium on in vitro propagation of Boswellia serrata Roxb. - Indian Journal of Biotechnology 10: 224-227.

[24] Werff, H. V. D., Richter, H. G. (1996): Towards an improved classification of Lauraceae. - Ann. Missouri Bot. Gard. 83: 409-418. 